Mathematical Research Letters 10, 787-798 (2002)

\title{
THE THETA-OPERATOR AND THE DIVISORS OF MODULAR FORMS ON GENUS ZERO SUBGROUPS
}

\author{
Scott Ahlgren
}

\section{Introduction and Statement of Results.}

If $f(z)$ is a meromorphic function with period 1 on the upper half-plane $\mathbb{H}$ with Fourier expansion of the form

$$
f(z)=\sum_{n=h}^{\infty} a(n) q^{n} \quad\left(q:=e^{2 \pi i z}\right),
$$

then we define the theta-operator by

$$
\theta f(z):=\frac{1}{2 \pi i} \frac{d}{d z} f(z)=\sum_{n=h}^{\infty} n a(n) q^{n} .
$$

This operator plays a fundamental role in the theory of modular forms, modular forms modulo $p$, and $p$-adic modular forms (see, for example, [Se], [Sw-D]). In a recent paper [B-K-O], Bruinier, Kohnen, and Ono have studied the action of the theta operator on meromorphic modular forms on $\mathrm{SL}_{2}(\mathbb{Z})$ in relation to the values of a certain sequence of modular functions at points $\tau$ in the divisor of $f$. Their description of this action leads to a number of consequences connected to the exponents in the infinite product expansions of modular forms, congruence properties and $p$-adic formulas for class numbers of imaginary quadratic fields, and recurrence relations for Fourier coefficients.

Throughout, we agree that $q:=e^{2 \pi i z}$, and, as usual, we denote by $E_{2}(z)$ the weight two Eisenstein series

$$
E_{2}(z):=1-24 \sum_{n=1}^{\infty} \sum_{d \mid n} d q^{n} .
$$

If $f$ is a meromorphic modular form on $\mathrm{SL}_{2}(\mathbb{Z})$, then it is well-known that there exists a meromorphic weight two modular form $f_{\theta}$ with the property that

$$
\theta f=\frac{k E_{2} f}{12}-f f_{\theta} .
$$

Received June 21, 2002.

The author thanks the National Science Foundation for its support through grant DMS 01-34577. 
Bruinier, Kohnen, and Ono show that $f_{\theta}$ can in fact be defined explicitly in terms of the values of a certain sequence of modular functions at points $\tau$ in the divisor of $f$; most of the consequences outlined in the last paragraph follow from this description.

It is natural to investigate analogues of this work for modular forms on more general subgroups; here we consider the problem for groups $\Gamma_{0}(p)$ with $p \in$ $\{2,3,5,7,13\}$. In particular, in analogy with (1.2), we are able to give an explicit formula for the action of the theta-operator on any modular form for these genus zero groups (see Theorem 2 below). As a consequence, we obtain formulas for the exponents of infinite product expansions and recurrence relations for Fourier coefficients of modular forms on these groups.

To state our results requires some notation. As usual, define Dedekind's etafunction by

$$
\eta(z):=q^{\frac{1}{24}} \prod_{n=1}^{\infty}\left(1-q^{n}\right) .
$$

Suppose that $p$ is one of $2,3,5,7$, or 13 . Then the genus of $\Gamma_{0}(p)$ is zero, and the function

$$
\phi_{p}(z):=\left(\frac{\eta(z)}{\eta(p z)}\right)^{\frac{24}{p-1}}
$$

is a univalent modular function on $\Gamma_{0}(p)$ with a simple pole at infinity and with a simple zero (as measured in local coordinates) at 0 . For these primes $p$, we define a sequence of modular functions $\left\{j_{n}^{(p)}(z)\right\}_{n=1}^{\infty}$ as follows: $j_{n}^{(p)}(z)$ is the unique modular function on $\Gamma_{0}(p)$ which is holomorphic on $\mathbb{H}$, which vanishes at the cusp 0 and whose Fourier expansion at infinity has the form

$$
j_{n}^{(p)}(z)=q^{-n}+c(0)+c(1) q+c(2) q^{2}+\ldots
$$

For $n=1$ we have

$$
j_{1}^{(p)}(z)=\phi_{p}(z)
$$

and it is clear for $n=2,3, \ldots$ that such functions can be constructed as monic polynomials in $\phi_{p}(z)$ with constant term equal to zero. To see that these conditions determine the functions $j_{n}^{(p)}$ uniquely, we note that that the difference of two functions satisfying (1.3) has no poles in a fundamental domain, and must therefore be constant. Since both forms vanish at 0 , this constant must be zero. As an example of this construction, we consider the case $p=5$. We then have

$$
\begin{array}{cc}
j_{1}^{(5)}(z) & =\phi_{5}(z)=q^{-1}-6+9 q+10 q^{2}-30 q^{3}+\ldots, \\
j_{2}^{(5)}(z) & =\phi_{5}(z)^{2}+12 \phi_{5}(z)=q^{-2}-18+20 q+21 q^{2}+192 q^{3}+\ldots \\
j_{3}^{(5)}(z) & =\phi_{5}(z)^{3}+18 \phi_{5}(z)^{2}+81 \phi_{5}(z)=q^{-3}-24-90 q+288 q^{2}+144 q^{3}+\ldots, \\
\vdots & \vdots
\end{array}
$$


As usual, we define the operator $V(p)$ by the map $z \mapsto p z$; in other words,

$$
\left(\sum_{n=h}^{\infty} a(n) q^{n}\right) \mid V(p):=\sum_{n=h}^{\infty} a(n) q^{p n} .
$$

Also, if $\tau \in \mathbb{H}$ and $p \in\{2,3,5,7,13\}$, then we define

$$
H_{\tau}^{(p)}(z):=\sum_{n=1}^{\infty} j_{n}^{(p)}(\tau) q^{n} .
$$

With this notation we can state the main results. Our first theorem gives an explicit formula for the functions $H_{\tau}^{(p)}$.

Theorem 1. If $p \in\{2,3,5,7,13\}$ and $H_{\tau}^{(p)}$ is as defined in (1.4), then

$$
H_{\tau}^{(p)}(z)=\frac{p \cdot E_{2}(z) \mid V(p)-E_{2}(z)}{p-1}\left(\frac{\phi_{p}(z)}{\phi_{p}(z)-\phi_{p}(\tau)}-1\right)
$$

In particular, $H_{\tau}^{(p)}$ is a weight two meromorphic modular form on $\Gamma_{0}(p)$.

We note, for the primes $p$ under consideration, that the modular form

$$
\frac{p \cdot E_{2} \mid V(p)-E_{2}}{p-1}=1+\frac{24}{p-1} \cdot q+\ldots
$$

forms a basis for the one-dimensional space $M_{2}\left(\Gamma_{0}(p)\right)$.

Our next theorem gives an explicit formula for the action of the theta operator on meromorphic modular forms for these subgroups $\Gamma_{0}(p)$. If $p$ is prime, then denote by $\mathcal{F}_{p}$ a fundamental domain for the action of $\Gamma_{0}(p)$ on $\mathbb{H}$. Our convention is that $\mathcal{F}_{p}$ does not include the two cusps $\infty$ and 0 ; in other words, $\mathcal{F}_{p}$ corresponds to the usual non-compact Riemann surface $Y_{0}(p):=\Gamma_{0}(p) \backslash \mathbb{H}$. If $\tau \in \mathbb{H}$ then define $\ell_{\tau}^{(p)} \in\{1,2,3\}$ by

$$
\ell_{\tau}^{(p)}:=\text { the order of the isotropy subgroup of } \tau \text { in } \Gamma_{0}(p) /\{ \pm I\} \text {. }
$$

If $\tau \in \mathbb{H}$, and $f$ is a modular form for $\Gamma_{0}(p)$, then we define

$$
v_{\tau}^{(p)}(f):=\frac{1}{\ell_{\tau}^{(p)}} \operatorname{ord}_{\tau}(f)
$$

where $\operatorname{ord}_{\tau}(f)$ denotes the standard order of vanishing of $f$ at the point $\tau \in \mathbb{H}$. Then $v_{\tau}^{(p)}$ measures order of vanishing with respect to local coordinates on $Y_{0}(p)$ (see [Sh, §2.4] for a complete discussion).

With this notation we can state the second theorem. 
Theorem 2. Suppose that $p \in\{2,3,5,7,13\}$, and that $f(z)=\sum_{n=h}^{\infty} a(n) q^{n}$ is a meromorphic modular form of weight $k$ for $\Gamma_{0}(p)$, normalized so that $a(h)=1$. Define the weight two modular form

$$
f_{\theta}(z):=\sum_{\tau \in \mathcal{F}_{p}} v_{\tau}^{(p)}(f) \cdot H_{\tau}^{(p)}(z)
$$

Then we have

$$
\frac{\theta f}{f}=-f_{\theta}+\frac{h-k / 12}{p-1} \cdot p E_{2} \mid V(p)+\frac{p k / 12-h}{p-1} \cdot E_{2}
$$

Our next theorem gives a formula for the coefficients in the infinite product expansion of any modular form on $\Gamma_{0}(p)$ with $p \in\{2,3,5,7,13\}$ (see Lemma 2.1 below for details regarding this expansion). As usual, we define

$$
\sigma(n):=\sum_{d \mid n} d
$$

and we agree that $\sigma(n):=0$ if $n \notin \mathbb{N}$.

Theorem 3. Suppose that $p \in\{2,3,5,7,13\}$, and that $f(z)=\sum_{n=h}^{\infty} a(n) q^{n}$ is a meromorphic modular form of weight $k$ on $\Gamma_{0}(p)$, normalized so that $a(h)=1$. Let $\{c(n)\}_{n=1}^{\infty}$ denote the complex numbers for which

$$
f=q^{h} \prod_{n=1}^{\infty}\left(1-q^{n}\right)^{c(n)}
$$

Then for each $n \geq 1$ we have

$$
\sum_{d \mid n} c(d) d=\sum_{\tau \in \mathcal{F}_{p}} v_{\tau}^{(p)}(f) j_{n}^{(p)}(\tau)+\left(\frac{24 h-2 k}{p-1}\right) p \sigma(n / p)+\left(\frac{2 p k-24 h}{p-1}\right) \sigma(n) .
$$

Equivalently, we have

$$
c(n)=\frac{1}{n} \sum_{d \mid n} \mu(n / d) \sum_{\tau \in \mathcal{F}_{p}} v_{\tau}^{(p)}(f) j_{d}^{(p)}(\tau)+ \begin{cases}(2 p k-24 h) /(p-1) & \text { if } p \nmid n, \\ 2 k & \text { if } p \mid n .\end{cases}
$$

As an application of these results, we prove a theorem which gives a recurrence for the Fourier coefficients of meromorphic modular forms

$$
f(z)=q^{h}+\sum_{n=h+1}^{\infty} a(n) q^{n}
$$


on the spaces $\Gamma_{0}(p)$ under consideration. For each $n \geq 1$, define the polynomial

$$
\begin{aligned}
\sum_{\substack{m_{1}+2 m_{2}+\cdots+(n-1) m_{n-1} \\
m_{1}, \ldots, m_{n-1} \geq 0}}(-1)^{m_{1}+m_{2}+\cdots+m_{n-1}} \frac{\left(m_{1}+\cdots+m_{n-1}-1\right) !}{m_{1} ! m_{2} ! \ldots m_{n-1} !} x_{1}^{m_{1}} \ldots x_{n-1}^{m_{n-1}} \\
+\frac{1}{n}\left(\frac{2 K-24 H}{p-1}\right) p \sigma(n / p)+\frac{1}{n}\left(\frac{24 H-2 p K}{p-1}\right) \sigma(n) .
\end{aligned}
$$

Then we have

Theorem 4. Suppose that $p \in\{2,3,5,7,13\}$ and that

$$
f(z)=q^{h}+\sum_{n=h+1}^{\infty} a(n) q^{n}
$$

is a meromorphic modular form of weight $k$ on $\Gamma_{0}(p)$. For each $n \geq 1$, let the polynomial $F_{n}^{(p)}\left(K, H, x_{1}, \ldots, x_{n-1}\right)$ be defined as in (1.9). Then we have

$$
a(h+n)=F_{n}^{(p)}(k, h, a(h+1), \ldots, a(h+n-1))-\frac{1}{n} \sum_{\tau \in \mathcal{F}_{p}} v_{\tau}^{(p)}(f) j_{n}^{(p)}(\tau) .
$$

The first several cases of Theorem 4 give the following formulas.

$$
\begin{aligned}
& a(h+1)=\frac{24 h-2 p k}{p-1}-\sum_{\tau \in \mathcal{F}_{p}} v_{\tau}^{(p)}(f) j_{1}^{(p)}(\tau) \\
& a(h+2)= \frac{1}{2} a(h+1)^{2}+\frac{3}{2}\left(\frac{24 h-2 p k}{p-1}\right)+\frac{1}{2}\left(\frac{2 k-24 h}{p-1}\right) p \sigma(2 / p)-\frac{1}{2} \sum_{\tau \in \mathcal{F}_{p}} v_{\tau}^{(p)}(f) j_{2}^{(p)}(\tau), \\
& a(h+3)=-\frac{1}{3} a(h+1)^{3}+a(h+1) a(h+2)+\frac{4}{3}\left(\frac{24 h-2 p k}{p-1}\right)+\frac{1}{3}\left(\frac{2 k-24 h}{p-1}\right) p \sigma(3 / p) \\
& \quad-\frac{1}{3} \sum_{\tau \in \mathcal{F}_{p}} v_{\tau}^{(p)}(f) j_{3}^{(p)}(\tau) \\
& a(h+4)=\frac{1}{4} a(h+1)^{4}+\frac{1}{2} a(h+2)^{2}-a(h+1)^{2} a(h+2)+a(h+1) a(h+3) \\
& \quad+\frac{7}{4}\left(\frac{24 h-2 p k}{p-1}\right)+\frac{1}{4}\left(\frac{2 k-24 h}{p-1}\right) p \sigma(4 / p)-\frac{1}{4} \sum_{\tau \in \mathcal{F}_{p}} v_{\tau}^{(p)}(f) j_{4}^{(p)}(\tau) .
\end{aligned}
$$

The formulas of Theorems 3 and 4 are of course simplest when the divisor of $f$ is supported at the cusps of $\Gamma_{0}(p)$. Many such forms can be constructed using the eta-function; for an example, we could take

$$
(p=5, k=0, h=-1): \phi_{5}(z)=\frac{\eta^{6}(z)}{\eta^{6}(5 z)}=q^{-1}-6+9 q+10 q^{2}-30 q^{3}+6 q^{4}-25 q^{5}+\ldots
$$


We also remark that these results can be extended to forms with Nebentypus (by applying the stated results to an appropriate power of the form in question). In the next section we prove Theorem 3 , and in the subsequent sections we use it to derive Theorems 1, 2, and 4. Our method follows roughly that of [B-K-O]; for convenience we work with differentials on the Riemann surface $X_{0}(p)$ instead of working directly with the relevant contour integrals. Adjustments must of course be made to handle the increased complexity of the fundamental domains $\mathcal{F}_{p}$. Finally, we mention that two recent papers (see $[\mathrm{At}],[\mathrm{C}-\mathrm{K}]$ ) consider similar problems with respect to certain other subgroups of $\mathrm{SL}_{2}(\mathbb{R})$.

\section{Proof of Theorem 3.}

We begin by stating a lemma whose proof may be found in [B-K-O, Prop. $2.1]$.

Lemma 2.1. Suppose that $f=\sum_{n=h}^{\infty} a(n) q^{n}$ is a meromorphic function in a neighborhood of $q=0$ and that $a(h)=1$. Then there are uniquely determined complex numbers $c(n)$ such that

$$
f=q^{h} \prod_{n=1}^{\infty}\left(1-q^{n}\right)^{c(n)},
$$

where the product converges in some neighborhood of $q=0$. Moreover, we have the identity

$$
\frac{\theta f}{f}=h-\sum_{n=1}^{\infty} \sum_{d \mid n} c(d) d q^{n} .
$$

In the next lemma we construct a modular form which will be important in what follows.

Lemma 2.2. Suppose that $p$ is prime and that $f$ is a meromorphic modular form of weight $k$ on $\Gamma_{0}(p)$. If $h$ is any constant, then the function

$$
F:=\theta f+\left\{\frac{k / 12-h}{p-1} \cdot p E_{2} \mid V(p)+\frac{h-p k / 12}{p-1} \cdot E_{2}\right\} \cdot f
$$

is a meromorphic modular form of weight $k+2$ on $\Gamma_{0}(p)$.

Proof of Lemma 2.2. If $\gamma=\left(\begin{array}{ll}a & b \\ c & d\end{array}\right) \in \mathrm{SL}_{2}(\mathbb{Z})$, then we have [Sch, p. 68] the transformation formula

$$
E_{2}(\gamma z)=(c z+d)^{2} E_{2}(z)-\frac{6 i c}{\pi}(c z+d) .
$$

Suppose for the duration of the proof that $\gamma=\left(\begin{array}{ll}a & b \\ c & d\end{array}\right) \in \Gamma_{0}(p)$. Then, since $p \gamma z=\gamma^{\prime}(p z)$, where $\gamma^{\prime}=\left(\begin{array}{cc}a & p b \\ c / p & d\end{array}\right)$, we see by (2.2) that

$$
E_{2}(p \gamma z)=(c z+d)^{2} E_{2}(p z)-\frac{6 i c}{p \pi}(c z+d) .
$$


From the fact that $f(\gamma z)=(c z+d)^{k} f(z)$, together with the definition (1.1), we find that

$$
(\theta f)(\gamma z)=(c z+d)^{k+2} \cdot \theta f(z)+\frac{k c}{2 \pi i}(c z+d)^{k+1} f(z)
$$

A computation using (2.2), (2.3), and (2.4) shows that, with $F(z)$ as defined in the statement of the lemma, we have

$$
F(\gamma z)=(c z+d)^{k+2} F(z) .
$$

The lemma follows.

As usual, we let $X_{0}(p)$ be the compact Riemann surface obtained by adjoining the two cusps 0 and $\infty$ to $Y_{0}(p)$. If $G(z)$ is a meromorphic weight two modular form on $\Gamma_{0}(p)$, then on the Riemann surface $X_{0}(p)$ we have the corresponding abelian differential $\omega_{G}:=G(z) d z$. A fundamental fact is that

$$
\sum_{Q \in X_{0}(p)} \operatorname{Res}_{Q} \omega_{G}=0
$$

(our convention will be that $\operatorname{Res}_{Q} \omega_{G}$ will denote the residue at $Q$ with respect to local coordinates on $X_{0}(p)$, while $\operatorname{Res}_{z=\tau} G(z)$ will denote the usual residue at a point $\tau \in \mathbb{H})$.

If $f$ is a function on $\mathbb{H}, k \in \mathbb{Z}$, and $\left(\begin{array}{ll}a & b \\ c & d\end{array}\right) \in \mathrm{GL}_{2}^{+}(\mathbb{R})$, then we define the operator $\left.\right|_{k}$ by

$$
\left.f(z)\right|_{k}\left(\begin{array}{cc}
a & b \\
c & d
\end{array}\right):=(a d-b c)^{k / 2}(c z+d)^{-k} f\left(\frac{a z+b}{c z+d}\right) .
$$

Suppose that $G$ has the following expansions at the cusps:

$$
\begin{aligned}
G(z) & =\sum_{n \geq n_{0}} a(n) q^{n} \quad \text { at } \infty, \\
\left.G(z)\right|_{2}\left(\begin{array}{cc}
0 & -1 \\
1 & 0
\end{array}\right) & =\sum_{n \geq n_{0}^{\prime}} b(n) q^{n / p} \quad \text { at } 0 .
\end{aligned}
$$

For $\tau \in \mathbb{H} \cup\{0, \infty\}$, let $Q_{\tau} \in X_{0}(p)$ be the point associated to $\tau$ under the usual identification. Then, computing with respect to local variables, we find that

$$
\operatorname{Res}_{Q_{\infty}} \omega_{G}=\frac{1}{2 \pi i} a(0), \quad \operatorname{Res}_{Q_{0}} \omega_{G}=\frac{p}{2 \pi i} b(0) .
$$

If $\tau \in \mathbb{H}$, then, with $\ell_{\tau}$ as defined in (1.5), a computation using local coordinates shows that

$$
\operatorname{Res}_{Q_{\tau}} \omega_{G}=\frac{1}{\ell_{\tau}} \operatorname{Res}_{\tau} G
$$


We now turn to the proof of Theorem 3; for the duration of this section we will fix a prime $p \in\{2,3,5,7,13\}$. For each $m \geq 1$, let $j_{m}(z)$ be the modular function defined in (1.3) (since $p$ is fixed, we will drop the superscript ${ }^{(p)}$ in our notation). Suppose that $f$ is a meromorphic modular form of weight $k$ on $\Gamma_{0}(p)$, and that, as in the statement of Theorem 3, we have

$$
f=q^{h} \prod_{n=1}^{\infty}\left(1-q^{n}\right)^{c(n)} .
$$

Then let $F(z)$ be the modular form of weight $k+2$ defined in Lemma 2.2 (in that Lemma we choose for $h$ the value given by (2.7)). We will apply the considerations of the last two paragraphs to the differential

$$
\omega_{m}:=\frac{F(z)}{f(z)} \cdot j_{m}(z) d z
$$

We begin by computing $\operatorname{Res}_{\infty} \omega_{m}$. By (1.3) we may write

$$
j_{m}(z)=q^{-m}+c_{m}(0)+O(q)
$$

with some constant $c_{m}(0)$. Using (2.1), the definition of $E_{2}(z)$, the description of $\frac{\theta f}{f}$ given in Lemma 2.1, and (2.5), we find that

$$
\begin{aligned}
2 \pi i \operatorname{Res}_{\infty} \omega_{m}=-\sum_{d \mid m} c(d) d+( & \left.\frac{24 h-2 k}{p-1}\right) p \sum_{d \mid \frac{m}{p}} d+\left(\frac{2 p k-24 h}{p-1}\right) \sum_{d \mid m} d \\
& +c_{m}(0) \cdot\left\{h+\frac{k p / 12-p h}{p-1}+\frac{h-k p / 12}{p-1}\right\} .
\end{aligned}
$$

Therefore

$$
2 \pi i \operatorname{Res}_{\infty} \omega_{m}=-\sum_{d \mid m} c(d) d+\left(\frac{24 h-2 k}{p-1}\right) p \sigma(m / p)+\left(\frac{2 p k-24 h}{p-1}\right) \sigma(m)
$$

We now claim that

$$
\operatorname{Res}_{0} \omega_{m}=0 \text {. }
$$

To establish (2.10), recall that $j_{m}(z)$ vanishes at 0 ; its expansion at 0 therefore has the form

$$
j_{m}(-1 / z)=c q^{\frac{1}{p}}+\text { higher order terms. }
$$

Define $w_{p}:=\left(\begin{array}{cc}0 & -1 \\ p & 0\end{array}\right)$. Using $(2.5),(2.8)$, and (2.11), we see that to prove $(2.10)$, it will suffice to show that

$$
\left.\frac{F}{f}(z)\right|_{2} w_{p} \text { has an expansion in non-negative powers of } q .
$$


For convenience, let $g(z):=\left.f(z)\right|_{k} w_{p}$. Since $g(z)$ is again a modular form of weight $k$ on $\Gamma_{0}(p)$, we conclude from Lemma 2.1 that we have an expansion of the form

$$
\frac{\theta g}{g}(z)=h^{\prime}+\sum_{n=1}^{\infty} \sum_{d \mid n} c^{\prime}(d) d q^{n}
$$

Now, since $g(z)=p^{-k / 2} \cdot z^{-k} f(-1 / p z)$, a computation using (1.1) shows that

$$
\left.\frac{\theta f}{f}(z)\right|_{2} w_{p}=\frac{\theta g}{g}(z)+\frac{k}{2 \pi i z} \text {. }
$$

By (2.2) we have

$$
z^{-2} E_{2}(-1 / z)=E_{2}(z)+\frac{12}{2 \pi i z}
$$

Therefore, we obtain

$$
\begin{gathered}
\left.E_{2}(z)\right|_{2} w_{p}=p E_{2}(p z)+\frac{12}{2 \pi i z} \\
\left.\left(E_{2}(z) \mid V(p)\right)\right|_{2} w_{p}=p^{-1} E_{2}(z)+\frac{12}{2 \pi i p z} .
\end{gathered}
$$

Using (2.1), (2.14), (2.15), and (2.16), we compute that

$$
\left.\frac{F}{f}(z)\right|_{2} w_{p}=\frac{\theta g}{g}(z)+\frac{k / 12-h}{p-1} \cdot E_{2}(z)+\frac{h-p k / 12}{p-1} \cdot p E_{2}(z) \mid V(p) .
$$

Our claim (2.12), and with it (2.10), now follows easily from (2.17) and (2.13).

We now compute the residue of $\omega_{m}$ at points $Q_{\tau} \in Y_{0}(p)$ corresponding to points $\tau \in \mathbb{H}$. Since $E_{2}(z)$ and $j_{m}(z)$ are holomorphic on $\mathbb{H}$, and since

$$
\frac{\theta f}{f}(z)=\frac{1}{2 \pi i} \frac{f^{\prime}}{f}(z)
$$

we find for $\tau \in \mathbb{H}$ that

$$
2 \pi i \operatorname{Res}_{z=\tau} \frac{F(z)}{f(z)} \cdot j_{m}(z)=2 \pi i\left(\operatorname{Res}_{z=\tau} \frac{\theta f}{f}(z)\right) \cdot j_{m}(\tau)=\operatorname{ord}_{\tau} f \cdot j_{m}(\tau)
$$

Using (2.6) and (1.6) we find that for $\tau \in \mathbb{H}$ we have

$$
2 \pi i \operatorname{Res}_{Q_{\tau}} \omega_{m}=v_{\tau}^{(p)}(f) \cdot j_{m}(\tau) .
$$

Using (2.9), (2.10), (2.18), the fact that the residues sum to zero, and the bijection between points $\tau \in \mathcal{F}_{p}$ and points $Q_{\tau} \in Y_{0}(p)$, we conclude that

$$
\sum_{d \mid m} c(d) d=\left(\frac{24 h-2 k}{p-1}\right) p \sigma(m / p)+\left(\frac{2 p k-24 h}{p-1}\right) \sigma(m)+\sum_{\tau \in \mathcal{F}_{p}} v_{\tau}^{(p)}(f) \cdot j_{m}(\tau) .
$$

This establishes (1.7). Formula (1.8) follows directly by Möbius inversion. 


\section{Proof of Theorem 2.}

Theorem 2 follows easily from Theorem 3 . If $f$ is as in Theorem 2 , then by Lemma 2.1, Theorem 3, and (1.4), we have

$$
\begin{aligned}
\frac{\theta f}{f} & =h-\sum_{\tau \in \mathcal{F}_{p}} v_{\tau}^{(p)}(f) H_{\tau}^{(p)}(z) \\
& +\left(\frac{h-k / 12}{p-1}\right)\left(-24 p \sum_{n=1}^{\infty} \sigma(n / p) q^{n}\right)+\left(\frac{p k / 12-h}{p-1}\right)\left(-24 \sum_{n=1}^{\infty} \sigma(n) q^{n}\right) \\
& =h-f_{\theta}+\left(\frac{h-k / 12}{p-1}\right) p E_{2} \mid V(p)+\left(\frac{p k / 12-h}{p-1}\right) E_{2}-\frac{h-k / 12}{p-1} \cdot p-\frac{p k / 12-h}{p-1} \\
& =-f_{\theta}+\left(\frac{h-k / 12}{p-1}\right) p E_{2} \mid V(p)+\left(\frac{p k / 12-h}{p-1}\right) E_{2} .
\end{aligned}
$$

This gives Theorem 2 .

\section{Proof of Theorem 1.}

To prove Theorem 1, we first apply Lemma 2.2 with $f(z)=\phi_{p}(z), k=0$, and $h=-1$. We find that

$$
G_{p}:=\frac{\theta \phi_{p}}{\phi_{p}}+\frac{p E_{2} \mid V(p)-E_{2}}{p-1}
$$

is a modular form of weight two on $\Gamma_{0}(p)$ with constant term 0 . Moreover, $G_{p}$ is holomorphic on $\mathbb{H}$ and at the cusps (the only thing which needs to be checked is holomorphicity at 0 ; this can be verified using an argument as in (2.12)-(2.17)). Since, for the values of $p$ under consideration, the spaces $M_{2}\left(\Gamma_{0}(p)\right)$ are onedimensional, and spanned by $\frac{p E_{2} \mid V(p)-E_{2}}{p-1}=1+\ldots$, we find that $G_{p}=0$, or in other words that

$$
\frac{\theta \phi_{p}}{\phi_{p}}=\frac{E_{2}-p E_{2} \mid V(p)}{p-1} .
$$

It follows that for $\tau \in \mathbb{H}$ we have

$$
\frac{\theta\left(\phi_{p}(z)-\phi_{p}(\tau)\right)}{\phi_{p}(z)-\phi_{p}(\tau)}=\frac{E_{2}-p E_{2} \mid V(p)}{p-1} \cdot \frac{\phi_{p}(z)}{\phi_{p}(z)-\phi_{p}(\tau)} .
$$

Finally, we apply Theorem 2 with $f(z)=\phi_{p}(z)-\phi_{p}(\tau)$. Since $f$ is a univalent function, we have the simple relation

$$
f_{\theta}(z)=H_{\tau}^{(p)}(z)
$$

Therefore, Theorem 2 gives

$$
\frac{\theta\left(\phi_{p}(z)-\phi_{p}(\tau)\right)}{\phi_{p}(z)-\phi_{p}(\tau)}=-H_{\tau}^{(p)}(z)+\frac{E_{2}(z)-p E_{2}(z) \mid V(p)}{p-1} .
$$

Theorem 1 follows upon comparing (3.1) and (3.2). 


\section{Proof of Theorem 4.}

Let $f$ be as given in the statement of Theorem 4, and write

$$
f(z)=q^{h}+\sum_{n=h+1}^{\infty} a(n) q^{n}=q^{h} \cdot \prod_{n=1}^{\infty}\left(1-q^{n}\right)^{c(n)}
$$

as in Lemma 2.1. We define

$$
b(n):=\sum_{d \mid n} c(d) d
$$

by Theorem 3 we have

$$
b(n)=\sum_{\tau \in \mathcal{F}_{p}} v_{\tau}^{(p)}(f) j_{n}^{(p)}(\tau)+\left(\frac{24 h-2 k}{p-1}\right) p \sigma(n / p)+\left(\frac{2 p k-24 h}{p-1}\right) \sigma(n) .
$$

From Lemma 2.1 we find that

$$
\left(q^{h}+\sum_{n=h+1}^{\infty} a(n) q^{n}\right)\left(h-\sum_{n=1}^{\infty} b(n) q^{n}\right)=h q^{h}+\sum_{n=h+1}^{\infty} n a(n) q^{n} .
$$

It follows that for $n \geq 1$ we have

$$
n a(h+n)=-b(1) a(h+n-1)-b(2) a(h+n-2)-\cdots-b(n) .
$$

In order to solve for $b(1), b(2), \ldots$ we use the fact that the recurrence (4.2) is essentially the same as that which relates the usual complete symmetric functions and power sums. The solution to this recurrence is well known (see, for instance, (2.11) and Example 20 in $\S 1.2$ of $[\mathrm{M}]$ ). In particular, for $n \geq 1$ we have

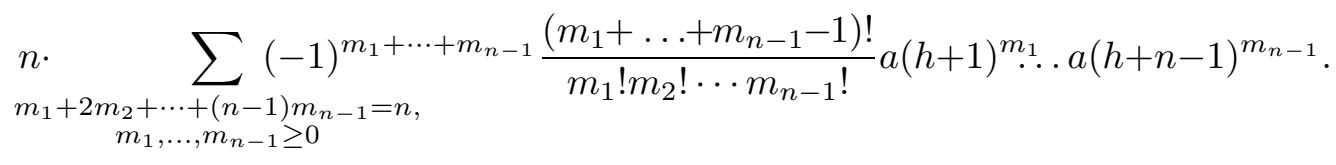

Theorem 4 now follows from (4.1), (4.3), and the definition (1.9).

\section{References}

[At] J. Atkinson, Divisors of modular forms on $\Gamma_{0}(4)$, preprint.

[B-K-O] J. Bruinier, W. Kohnen, and K. Ono, The arithmetic of the values of modular functions and the divisors of modular forms, Compositio Math. (to appear).

[C-K] Y. Choie and W. Kohnen, Special values of modular functions on Hecke groups, Abh. Math. Sem. Hamburg (to appear). 
[M] I. G. Macdonald, Symmetric functions and Hall polynomials, Second Edition, Oxford University Press, Oxford, 1995.

[Sch] B. Schoenberg, Elliptic modular functions, Springer-Verlag, New York, Heidelberg, Berlin, 1970.

[Se] J.-P. Serre, Formes modulaires et fonctions zêta p-adiques, Springer Lect. Notes in Math. 350, (1973), 191-268.

[Sh] G. Shimura, Introduction to the arithmetic theory of automorphic functions, Princeton University Press, 1971.

[Sw-D] H. P. F. Swinnerton-Dyer, On $\ell$-adic representations and congruences for modular forms, Springer Lect. Notes in Math. 350, (1973), 1-55.

Department of Mathematics, University of Illinois, Urbana, Illinois 61801

E-mail address: ahlgren@math.uiuc.edu 